\title{
Editorial
}

\section{Genetic re-targeting of T lymphocyte specificity}

Throughout history new strategies for the treatment of cancer and infectious diseases have primarily been based on discoveries in basic biology. Our deepening understanding of the immune system at the cellular and molecular level, together with the advances in immune- and molecular biotechnologies open new avenues for the design of novel forms of immuno-gene therapy.

Today, no clinical therapy gives such clear-cut cure, especially in the case of solid tumors, as the surgeon's knife. It is radical and has precision. However, tumors are not always surgically accessible or excisable. The same is true for virally infected cells. Therefore, immunology-based therapeutic strategies aim at creating welldefined target cell specificities for the treatment of cancer or viral infections. Target specificities are guaranteed by virtue of the expression of specific receptors on $\mathrm{T}$ - and B-lymphocytes, the T cell receptors (TCRs) and antibody $(\mathrm{Ab})$ receptors, respectively. When such target-specific lymphocytes (or their products) can be generated in sufficient numbers ex or in vivo, an immune attack on and elimination of the harmful target cells occurs and leaves normal tissues intact. This idea goes back to the beginning of the 20th century when Paul Ehrlich put forward the 'magic bullet' hypothesis, in which the immune system is identified as the surveillance mechanism to arrest cancer cells or to eradicate virus-infected cells. ${ }^{1}$ At the end of the 19th century, William Coley used anti-cancer 'vaccines' consisting of bacteria to combat cancer. The first wave of immunotherapy started rolling: for six decades thereafter cancer patients underwent non-specific immune-stimulation by administration of relatively crude leukocyte extracts such as transfer factor, immune RNA, bacterial extracts, levamisole and Coley's toxin. This happened in spite of the fact that little was known about the various components of the immune system. Even less was known about the existence of tumor associated antigens (TAA). In 1970, Burnett reformulated the immunosurveillance theory, emphasizing that cellmediated specific immunity played the critical role in the surveillance and control of cancer and infectious diseases. $^{2}$ This triggered the call for better defined and highly purified biological response modifiers (BRM), including immune-specific $\mathrm{T}$ lymphocytes and monoclonal (m)Abs.

In the 1980s, the second wave of 'specific' immunotherapy started to roll, now with the emphasis on clinical adoptive transfer of in vitro activated and expanded $\mathrm{T}$ lymphocytes made possible by the advent of new biotechnologies, eg production of $\mathrm{mAbs}$ and in vitro expansion of immune cells. These $\mathrm{T}$ lymphocytes not only have the capacity to kill tumor and virus-infected target cells directly, but also to produce lymphokines which, through their immuno-regulatory function, recruit other target-cell-specific immune functions. The ultimate goal of all these efforts was and is to generate a targetcell-specific and long-lasting immune response that not only eradicates the bad guys but also provides protection for future encounters. A host of immune effector cells such as natural killer (NK) cells, macrophages, antigenpresenting dendritic cells, B- and T-lymphocytes, as well as their products, signalling cytokines, operate in an orchestrated fashion. The immune effector mechanisms range from direct target cell cytolysis to cytostasis.

Synergism creates novelty.

Gene cloning and viral vector-mediated gene transfer, as well as the specific targeting of immune cells and viral vectors, and the concerted and scaled-up use of these technologies provide us with the tools for immunogene therapy.

T lymphocytes and Abs display exquisite specificities for their target antigens. The wide diversity of TCR and immunoglobulin (Ig) specificities are the key characteristics of the immune system. It is created by the process of germ-line rearrangements involving the variable (V), diversity (D) and for Ig-heavy chain $(\mathrm{H})$, and joining $(\mathrm{J})$ gene segments. Only in the case of $\mathrm{Ig}$ do somatic mutations add to the antigen diversity. In short the $\mathrm{V}, \mathrm{D}$ and J segments are combined to form the antigen binding domains. Cloning of the antigen-binding regions allows direct linkage of these regions to a signalling element, eg the $\zeta$-chain derived from TCR complexes, in order to form a chimeric receptor. T lymphocytes do not only show exquisite antigen specificity but also have a capacity to destroy their targets that can compete with surgical efficacy. After all, without immuno-suppression organ transplants are totally rejected. It is the daily routine of the immune system that protects us against continuous viral and bacterial attacks throughout life. In cases where tumors arise and express a tumor-specific rejection antigen, as is the case in chemically induced or virus-induced tumors, immune-specific $\mathrm{T}$ lymphocytes efficiently and totally eradicate established tumors in animals models. Unfortunately, most clinically diagnosed human tumors lack such immunogenic, tumor-specific rejection antigens. Consequently, it is very difficult to identify, isolate and expand human tumor-specific T lymphocytes in order to use them as therapeutic tools. Nevertheless, human tumor-infiltrating lymphocytes (TIL) can be isolated which specifically interact with tumor cells in 
vitro, resulting in tumor cell lysis. These TIL are also capable of causing tumor regression in vivo. These tumor regression antigens recognized by the TIL are presented by and restricted in the context of the major histocompatibility complex (MHC) class I antigens and may be shared among tumor cells of distinct histogenetic origins. Human tumor regression antigens have been identified on melanoma, breast, colon, ovarian and lymphomas (eg MART-1, gp100, tyrosinase, P15, TRP-1, $\beta$-catenin), and are not only found on the tumor cells, but also on normal tissues: they are differentiation antigens. These functions may, on the one hand, explain the difficulty in obtaining rejection antigen-specific $\mathrm{T}$ lymphocytes from cancer patients because our immune system is tolerant to them. On the other hand, it may explain why such T lymphocytes do not cause autoimmune reactions when infused into patients: apparently only tumor cells which show 'aberrant' expression of these TAA are attacked by these cytotoxic T lymphocytes (CTL). Under certain conditions the tolerance against the differentiation antigens can be broken and specific immune T lymphocytes subsequently appear to develop into CTL. Such CTL or their precursors can now be identified, isolated and manipulated in the laboratory. Also a range of $\mathrm{mAbs}$ is available which recognize TAA in a MHC-unrestricted fashion. The success rate of generating and isolating tumor-specific CTL is, however, too low to allow wide clinical application, with the notable exception of CTL therapy of melanoma and of EBV-transformed B lymphoproliferations.

One way to broaden the clinical application is to clone the tumor antigen-binding regions of mAbs and TCRs already identified and isolated. Single chain (sc) or twochain chimeric receptors comprising a signal-transducing element can then be constructed. These tumor-specific chimeric receptors can be specifically transferred to CTL using retroviral vectors. Combined use of technologies to generate TAA-specific mAbs, to clone $\mathrm{Ab}$ or TCR antigen-binding regions specific for tumor cells, and specific retargeting of the specificity of vectors and human T lymphocytes now become clinically exploitable. We are to explore the possibilities of 'immunogene surgery'.

Retroviral vectors are widely used for gene transfer to somatic cells, including tumor cells and immune cells. Because they are relatively stable they transmit genes to the recipient cell and its progeny. No general approach, however, is available for in vivo target cell-specific gene transfer. Therefore, ex vivo gene transfer to recipient cells, including $\mathrm{T}$ lymphocytes, is applied in the majority of clinical protocols. In view of the relatively low efficiency of gene transfer to human $\mathrm{T}$ lymphocytes, additional expansion of gene modified $\mathrm{T}$ lymphocytes is required to reach 'therapeutic' cell numbers. Obviously in vivo administration of viral vectors for gene transfer would broaden its clinical application. It requires the availability of a general targeting approach which forces the vectors only to adhere to and transduce genes to selected target cells, with high efficacy. Two conditions need to be met. First, the target cells need to express a selective marker on their surface which specifically interact with a corresponding receptor, engineered to be expressed on the viral vector. Second, this target cell selective marker must allow efficient entry of the virus into the recipient cell for functional gene delivery.

For viral vectors, many attempts have been made to reshape the envelope glycoprotein or to substitute its receptor binding domain. These manipulations generally resulted in severely decreased gene transfer efficiencies. Probably the delicate balance which requires that the fusion peptide of the virus remains hidden until the virion has bound to the cell surface receptor is disturbed and inappropriate fusion is triggered. Single chain antibodies (scFvAb) have successfully been employed to target viral vectors to recipient cells., ${ }^{3,4,6}$ Spleen necrosis virus (SNV)-derived vectors appear to result in superior gene transduction to recipient cells when compared with murine leukemia virus (MLV)-derived vectors, probably because SNV enters the cell by direct membrane fusion within less than $5 \mathrm{~min}$ after binding to the receptor. Very recently the first proof of concept for a general approach of targeted gene transfer has been delivered.

Following immunization of mice with human T lymphocytes, a library of $\mathrm{scFv}$ display phages were prepared by cloning and fusion of cDNAs encoding the rearranged variable heavy and light chain domains of murine immunoglobulins. The respective $\mathrm{scFv}-\mathrm{cDNAs}$ were inserted into a phagemid resulting in the production of soluble scFv-displaying phages. Phages specifically binding to the cell surface were selected by panning. Single cDNAs of selected $\mathrm{scFv}$ were then inserted in a SNVderived env expression encoding vector to replace the env gene region coding for the SNV-derived surface (SU) protein. For subsequent selection of pTC-scFv-positive cells, the resistance gene zeo was also inserted into the plasmid. About 150 monoclonal retroviral vectors, each displaying a single cell-surface binding $\mathrm{scFv}$ in addition to wild-type SNV-Env, were generated by transient transfection of a packaging cell line. With six of the vector-containing supernatants $\mathrm{T}$ lymphocyte-specific gene transfer was repeatedly obtained (Engelstädter and Cichutek, personal communication).

The strength of this targeting strategy is that it operationally selects first for the target cell specific vectors and second for the functional combinations of $\mathrm{scFv}$ expressed on the vector and target cell selective ligands facilitating functional gene delivery. This novel approach circumvents the need to understand fully all targeting and fusion events in order to develop efficient and specific gene transduction technologies. General application of this virus targeting principle critically depends on whether indeed other candidate recipient cells express unique ligands which, at the same time, facilitate functional virus entry for gene delivery.

As detailed earlier, scFvAb type receptors comprise the $\mathrm{V}$ domains of both the heavy and the light chain of an antibody molecule connected by a peptide bridge. The peptide bridge is encoded in the spatial region inserted between the coding regions of the two $\mathrm{V}$ domains. ${ }^{5}$ Human T lymphocytes can now be endowed with predefined Ab-type specificities and make clinical application possible. ${ }^{6}$ Several groups reported on this specific targeting of primary human $\mathrm{T}$ lymphocytes with scFvAb. The low success rate, however, may appear not only to depend on the type of viral vector used, but also on the gene structure of the $\mathrm{scFvAb}$. In addition to retargeting viral vectors with high gene transfer efficiency, our attention should focus on the development of a general prin-

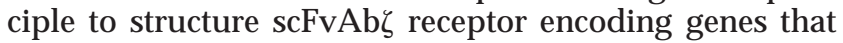
allow their expression on the membrane of CTL, whilst retaining antigen binding and signalling capacity. The therapeutic potential of T lymphocytes grafted with clini- 
cally relevant scFvAbs, and possibly TCRs, is enormous. Retargeted lymphocytes can then be used to combat cancer and viral infections. Clinical targets comprise the prophylaxis of CMV reactivation following bone marrow transplantation, the (prophylactic) treatment of the EBVtransformed B lymphoproliferations, elimination of autoimmune T lymphocytes, and the treatment of solid and fluid tumors. Although limited in number, in some of these areas adoptive transfer of immune $\mathrm{T}$ lymphocytes already have shown clinical efficacy. The third wave of immunogene therapy with surgical power is just about to start rolling.

RLH Bolhuis and JW Gratama Department of Clinical and Tumor Immunology

Daniel den Hoed Cancer Center Groene Hilledijk 301

Rotterdam, The Netherlands

\section{References}

1 Ehrlich P. Ueber den Jetzigen Stand der Karzinomforschung. Ned Tijdsch Geneeskd 1909; 35: 273-290.

2 Burnett FM. The concept of immunological surveillance. Progr Exp Tumor Res 1970; 13: 1-27.

3 Dornberg R. From the natural evolution to the genetic manipulation of the host range of retroviruses. Biol Chem 1997; 378: 457-468.

4 Cosset F-L, Russell SJ. Targeting retrovirus entry. Gene Therapy 1996; 3: 946-956.

5 Bird RE et al. Single-chain antigen-binding proteins. Science 1988 242: 423-426.

6 Eshhar Z, Waks T, Gross G, Schindler DG. Specific activation and targeting of cytotoxic $\mathrm{T}$ lymphocytes through chimeric single chains consisting of antibody-binding domains and the $\gamma$ or $\zeta$ subunits of the immunoglobulin and T cell receptors. Proc Natl Acad Sci USA 1993; 90: 720-724. 